\title{
Teoría de las representaciones sociales. Una aproximación al estado del arte en América Latina
}

\section{Approach towards a state of the art on theory of social representations in Latin America}

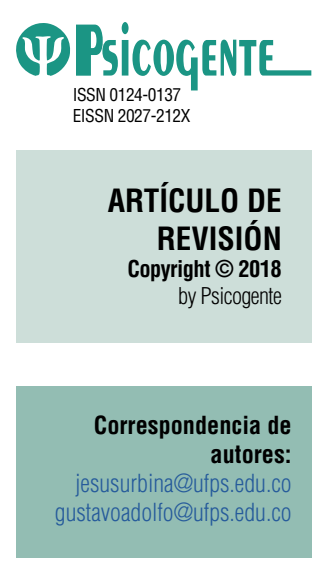

Recibido: 01-02-17 Aceptado: $04-12-17$ Publicado: 01-07-18

Jesús Ernesto Urbina Cárdenas (D) - Gustavo Adolfo Ovalles Rodríguez iD
Universidad Francisco de Paula Santander, Bogotá, Colombia

Resumen

Objetivo: Realizar una aproximación al estado del arte de las investigaciones en representaciones sociales en América Latina.

Método: Investigación de tipo documental que consideró las siguientes fases: preoperatoria, heurística y hermenéutica. La información, correspondiente a 172 artículos de investigación empírica y 102 artículos de naturaleza teórica, fue clasificada mediante la construcción de bases de datos y matrices en la Hoja de Cálculo Excel.

Resultados: Los trabajos de investigación más relevantes han sido desarrollados en países como Brasil, México, Venezuela y Argentina, los cuales están principalmente orientados por el enfoque procesual y se enfocan principalmente en áreas temáticas como salud, género, problemáticas sociales, educación, política y cultura.

Conclusiones: En América Latina se han realizado aportes significativos en el campo de la Teoría de las Representaciones Sociales como enfoque investigativo de las Ciencias Sociales y Humanas. Con un predominio del enfoque procesual, los estudios se orientan hacia la comprensión y transformación de la realidad social.

Palabras clave: representaciones sociales, enfoques teóricos, metodologías.

Abstract

Objective: this review paper aims to submit an approach towards a state of the art on social representations in Latin America. It shows an overview in relation with the Theory of Social Representations' contribution as a research topic of Social Human Sciences.

Method: This research was conducted through a documentary review, classified by the creation of databases and matrices in the Worksheet Excel, as follows: 172 empirical research articles and 102 theoretical articles: considering the following phases: preoperative, heuristic and hermeneutic.

Results: The most relevant research projects mainly oriented by the approach process and focus on fields such as health, gender, social problems, education, politics and culture, have been developed in countries such as Brazil, Mexico, Venezuela and Argentina.

Conclusions: In Latin America, the Theory of Social Representations as a research approach has made significant contributions in Social and Human Sciences field. Toward the understanding and transformation of social reality, the studies are conducted suggesting a predominance of the processual approach.

Key Words: social representations, theoretical approaches, methodologies

Cómo citar este artículo (APA):

Urbina Cárdenas, J. E. \& Ovalles Rodríguez, G. A. (2018). Teoría de las representaciones sociales.Una aproximación al estado del arte en América Latina .

Psicogente 21(40), 495-544. https://doi.org/10.17081/psico.21.40.3088 


\section{INTRODUCCIÓN}

La Teoría de las Representaciones Sociales ha alcanzado un notable nivel de desarrollo en el ámbito de las Ciencias Sociales. Esta propuesta teórica e investigativa, caracterizada por su condición de transversalidad con otras disciplinas como la antropología, sociología y psicología cognitiva, posee una enorme aplicabilidad en la comprensión e interpretación de los diversos fenómenos sociales y humanos presentes en la realidad de cualquier contexto cultural.

Precisamente, una de las principales características de una representación social es la utilidad social (Rateau y Monaco, 2013). En este sentido, la construcción e interpretación de la realidad, a partir de una visión común dada por la representación, orienta las prácticas y acciones de los grupos y conglomerados humanos. Develar el significado y sentido de estas prácticas y sus consecuencias es el objetivo del investigador de las Ciencias Sociales y Humanas que asume las representaciones sociales como referente investigativo. Para Banchs y Lozada (2000), el conocimiento y análisis de una representación social constituye un paso significativo para la transformación de las prácticas sociales. Por su parte, Jodelet (2011) destaca la aplicación de esta teoría en la investigación de fenómenos complejos, relacionados con la evolución de la sociedad global y la diversidad de niveles desde los que pueden ser abordados.

Aunque la Teoría de las Representaciones Sociales apareció en el año 1961 con la publicación de la Tesis Doctoral de Serge Moscovici, El Psicoanálisis, su imagen y su público, es solo hasta 1980 que se inicia el desarrollo de estudios bajo la orientación de esta perspectiva de investigación, primero en Europa y posteriormente en América Latina. Estos trabajos han sido orientados por diversas aproximaciones teóricas con fundamentos ontológicos y epistemológicos claramente definidos y diferenciados que hacen de la Teoría de las Representaciones Sociales una teoría flexible y adaptable para el estudio de las diferentes problemáticas sociales y culturales (Rateau y Monaco, 2013).

No obstante, los tres principales enfoques u orientaciones teóricas de las Representaciones Sociales son los siguientes:

1. El modelo socio-genético, también conocido como enfoque procesual, desarrollado especialmente por Jodelet (2000); Wagner (1994a, 1994b, 1995, 1996, 1999, 2005); Markovà (1987, 2000, 2003); Jovchelovitch 
(2001, 2007); y la mayoría de los investigadores latinoamericanos como Arruda, Reigota, Guerrero, Banchs, Lozada, entre otros.

2. El modelo estructural, también conocido como la Escuela de Aix-enProvence, que se fundamenta en la Teoría del Núcleo Central y dentro del cual se pueden mencionar investigadores como Abric (2001), Flament (2001), Moliner (1989, 1993), Guimelli (1992), Rouquette (1994, 2010), Pereira de Sá (1998, 2000), entre otros.

3. El modelo socio-dinámico o de toma de posición, también identificado con la Escuela de Ginebra, liderado por Doise (1991a, 1991b, 1993, 2005) y otros investigadores como Clèmence (2005), Lorenzi-Cioldi (1994, 1996), Mugny y Carugati (1985), entre otros.

La mayor parte de las investigaciones han sido realizadas en Europa, especialmente en países como el Reino Unido, Austria e Italia y optan por el enfoque estructural. En América Latina, se destacan las investigaciones realizadas en Brasil, México, Venezuela y Argentina, las cuales están principalmente orientadas por el enfoque procesual (Araya, 2002).

El objeto de estudio de las investigaciones sobre representaciones sociales es amplio y presenta una gran diversidad. Ha sido reseñado por investigadores como Castorina, Farr y Abric (Alfonso, 2006). Se pueden mencionar al respecto: psicoanálisis, ciencia, salud y enfermedad, cuerpo humano, infancia, relaciones entre grupos humanos, educación, género, inteligencia, problemáticas sociales y políticas, etc.

Ahora bien, la construcción de un estado del arte en representaciones sociales significa un reto considerable teniendo en cuenta la elevada producción de investigaciones teóricas y empíricas que existen a nivel mundial e incluyen artículos, libros, capítulos de libros, ponencias en eventos, tesis de maestría y doctorado. Por lo tanto, se hace necesario tomar decisiones concernientes a la limitación del universo en estudio, definir las estrategias de búsqueda, selección e interpretación de la información, así como la orientación y redacción del documento final.

El presente documento no pretende, entonces, en forma alguna hacer una elaboración completa y rigurosa del estado del arte de las investigaciones en representaciones sociales. Su única finalidad es presentar los avances más significativos logrados en América Latina, específicamente en países como México, Brasil, Venezuela y Argentina. De esta manera, se espera que el lector tenga un panorama amplio de lo que ha sido la producción investi- 
gativa sobre el tema en nuestro continente; en otras palabras, se trata de una propuesta de aproximación al conocimiento generado en más de 30 años de trabajo continuo en el análisis de la realidad social y cultural, una notable contribución de las Ciencias Sociales para la construcción de un mundo más racional, más justo, y ante todo más humano.

\section{MÉTODO}

\subsection{Diseño}

Se desarrolló una investigación documental cuyo objetivo principal consistió en construir el estado del arte de las investigaciones fundamentadas en la Teoría de las Representaciones Sociales en América Latina. El diseño de la investigación implicó la búsqueda, clasificación, sistematización y el análisis de documentos relacionados con esta temática en el campo de las Ciencias Sociales y Humanas.

\subsection{Participantes}

Fueron considerados un total de 172 artículos de investigación empírica y 106 artículos de revisión o desarrollos teóricos.

\subsection{Instrumentos}

Para clasificar la información correspondiente a las investigaciones empíricas y teóricas, se construyeron bases de datos o matrices en EXCEL según categorías de análisis que se precisarán más adelante.

\subsection{Procedimientos}

El trabajo se desarrolló en las siguientes etapas:

a. Fase preoperatoria: Comprendió la definición del tema o problema objeto de estudio y de las palabras clave (descriptores).

b. Fase heurística: Durante esta se definieron los lugares y criterios de búsqueda.

c. Fase hermenéutica: Abarcó la clasificación, procesamiento y análisis de la información.

d. Redacción de un artículo de revision: Sobre el estado del arte en la investigación en representaciones sociales

Durante la fase preoperatoria se definió como objeto de estudio las investigaciones realizadas desde la perspectiva de la Teoría de las Representaciones Sociales en el ámbito de las Ciencias Sociales y Humanas. 
En la fase heurística se estableció como criterio de búsqueda al conjunto de los artículos académicos relacionados con investigaciones en representaciones sociales. Este proceso implicó la exploración de la web visible, así como de la invisible o profunda. La denominación web invisible o profunda hace referencia a todos los contenidos que no son accesibles mediante los motores de búsqueda tradicionales (Colombia Digital, 2014). A continuación se presenta un listado de los lugares visitados:

- Google Académico

- Webs especializadas como FLACSO, OEI (Revista Iberoamericana de Educación)

- Bases de datos: PROQUEST, DIALNET, REDALYC, SCIENCE DIRECT, SCIELO, SCOPUS

En la fase hermenéutica, se procedió primero a clasificar la información en artículos de investigación empírica (172 en total) y artículos de revisión o desarrollos teóricos (106 en total). Los estudios empíricos fueron ordenados de acuerdo con las siguientes categorías temáticas, las cuales surgieron como resultado del proceso de búsqueda: Ciencia y saber académico; salud y enfermedad; etapas del desarrollo humano (infancia, adolescencia y vejez); estudios de género; educación; trabajo; sociedad-política-economía (democracia, pobreza, violencia, etc.); comunidades humanas (inmigración, diversidad cultural, medioambiente).

\subsection{Análisis de resultados}

Para los artículos de investigación empírica encontrados se consideraron las siguientes categorías de análisis: campo temático, año, país, autor, título, tipo de investigación, objetivos, metodología, referentes teóricos, descripción de la investigación con sus resultados. En lo referente a los artículos de revisión o desarrollo teórico las categorías fueron las siguientes: año, país, autor, título, sinopsis de la investigación con sus principales alcances.

Cabe decir que la producción investigativa más relevante en términos porcentuales corresponde a los siguientes países: Brasil (12\%), Argentina (28\%), México (42\%) y Venezuela (18\%). En cuanto a las categorías temáticas se obtuvieron los siguientes resultados: Ciencia y saber académico (8\%), Comunidades humanas (9\%), Desarrollo humano (11 \%), Educación (20 \%), Salud y enfermedad (14\%), Trabajo (8 \%) y Sociedad-Política-Economía (30 \%). 


\subsection{Criterios de inclusión y exclusión}

Como criterios de inclusión se tuvieron en cuenta los descriptores: "representaciones sociales" y combinaciones como "enfoque procesual", "enfoque estructural" y "enfoque socio-dinámico". Se consideraron publicaciones académicas relacionadas con investigaciones de naturaleza empíricas o teóricas desarrolladas en América Latina; asimismo, se incluyeron trabajos que abarcan el amplio campo del objeto de estudio de las representaciones sociales, el cual fue mencionado en la descripción de la fase hermenéutica de la investigación.

No se tuvieron en cuenta las investigaciones que no hacían referencia concreta a la Teoría de las Representaciones Sociales, como tampoco aquellas que no se publicaron en revistas indexadas.

\section{RESULTADOS}

\subsection{Investigaciones realizadas en América Latina}

La difusión de los trabajos investigativos realizados en Europa permitió la expansión de la Teoría de las Representaciones Sociales hacia el continente latinoamericano, un territorio poseedor de una enorme riqueza cultural y con realidades sociales y humanas muy particulares, que en su dinámica evolutiva generan diversas problemáticas y fenómenos dignos de objeto de estudio para los investigadores de las Ciencias Sociales.

En este sentido, un punto de gran interés es la consideración de una psicología social latinoamericana, propuesta formulada por diversos autores y que Jodelet (2000) reitera enunciando sus características: cuestionamiento de las identidades nacionales y de los sistemas de valores, importancia de los contextos sociales y económicos, comprensión de los retos formulados por los procesos de modernización y globalización a los países en vía de desarrollo $y$, finalmente, el compromiso ético con la transformación de la existencia de los grupos humanos.

Jodelet (2000) reconoce al respecto que Latinoamérica ha sido una tierra fértil para el desarrollo de investigaciones en representaciones sociales. Además, existe una afinidad intelectual entre investigadores latinoamericanos $y$ europeos, debido a una preocupación común: la comprensión de realidades socioculturales inmersas en un tiempo histórico y cargadas de simbolismos que definen y organizan la existencia social. Justo por esta razón, ese trabajo intelectual lleva implícita una dimensión ética de gran valor que se manifiesta 
en el respeto y reconocimiento de la realidad del sujeto investigado y ante todo en la postura axiológica asumida por el investigador.

Cuatro países latinoamericanos destacan por su aporte en el desarrollo de investigaciones teóricas y empíricas en representaciones sociales: México, Brasil, Venezuela y Argentina. Las temáticas de los estudios son muy variadas y demuestran la gran aplicabilidad de la teoría en el vasto escenario de los fenómenos sociales y humanos: salud y enfermedad, educación, ciencia, política, sociedad, economía, medioambiente, género, trabajo, inmigración, etapas del desarrollo humano, entre otras. Esta diversidad de objetos de estudio es consecuencia de la flexibilidad y heterodoxia que presenta la Teoría de las Representaciones Sociales, virtudes que le otorgan un gran valor y que ya fueron citadas en la introducción del presente estado del arte (Banchs, 2000).

Enfocando situaciones concretas, en México, la Red Nacional de Investigadores en Representaciones Sociales y el Centro Mexicano para el Estudio de las Representaciones Sociales (CEMERS) fueron creados con el apoyo académico y científico de Moscovici. Su misión fundamental es promover la investigación en representaciones sociales como herramienta teórico-metodológica para el análisis de las diversas problemáticas de la sociedad mexicana. Un listado de investigadores relevantes incluye a Silvia Valencia, Silvia Domínguez, Silvia Gutiérrez, María Estela Ortega, María Isabel Arbezú, Tania Rodríguez, Yazmín Cuevas, Alfredo Guerrero, Francisco Uribe, Eulogio Romero, Juan Manuel Piña y Raúl Calixto.

Alfredo Guerrero es profesor de la Facultad de Psicología de la Universidad Nacional Autónoma de México (UNAM). Su actividad investigativa se ha enfocado en lo social, educativo y laboral, de modo que sus estudios cubren diversas áreas, pero especialmente se interesa en lo social y en la cultura política. En el año 2000, junto con Denise Jodelet editaron Develando la cultura (Jodelet y Guerrero, 2000), obra que reúne una serie de investigaciones sobre representaciones sociales realizadas por investigadores latinoamericanos como Ángela Arruda, María Auxiliadora Banchs, Mireya Lozada, Claritza Prado de Souza, Silvia Valencia y Francisco Uribe.

En la presentación del citado libro, Jodelet reconoce la importancia del trabajo conjunto de Europa y Latinoamérica en el campo de las representaciones sociales y menciona como punto común entre sus investigaciones y las de nuestro continente la aproximación metodológica cualitativa. Por su parte, 
Alfredo Guerrero participa con el estudio "La noción de igualdad en la cultura mexicana", en el que presenta los resultados de dos trabajos que indagan en la transformación que ha sufrido la representación social del concepto de "igualdad" en la sociedad mexicana después de la llamada Conquista. Para Guerrero (2000), la igualdad es un término controversial, relacionado con un debate universal que forma parte de los procesos comunicativos de los grupos sociales y se difunde a través de los mass media.

Silvia Domínguez, por otra parte, es profesora del Centro Universitario de Ciencias de la Salud de la Universidad de Guadalajara. Sus intereses investigativos son: la epistemología y metodología de las ciencias sociales, educación, representaciones sociales, percepción pública de la ciencia y comunicación social. Su trabajo "Significados de la ciencia en estudiantes universitarios. Aproximaciones a las representaciones sociales de la ciencia, del científico y de la actividad científica" (Domínguez, 2012) tiene como objetivo la comprensión del significado de la ciencia en los estudiantes de pregrado en Ciencias de la Salud de la Universidad de Guadalajara. Los resultados muestran un núcleo figurativo de la representación conformado por la finalidad de la ciencia como componente principal; la ciencia es percibida mediante una gran diversidad de significados, pero sobresale la percepción de su utilidad para explicar los fenómenos, comprender la realidad y resolver problemas que conducen al beneficio de la sociedad.

Tania Rodríguez Salazar, docente del Departamento de Estudios de la Comunicación Social de la Universidad de Guadalajara, orienta su actividad investigativa principalmente hacia la sociología de la cultura, la sociología de las emociones y las representaciones sociales. Sus publicaciones incluyen libros, capítulos de libros, artículos de revisión teórica y artículos de investigación empírica. En el año 2007, junto con María Lourdes García, coordinó la publicación de Representaciones Sociales. Teoría e Investigación (Rodríguez y García, 2007), obra que recopila trabajos de investigación teórica y empírica de investigadores de renombre en Europa y Latinoamérica como Denise Jodelet, María Auxiliadora Banchs, Pascal Moliner, José Valencia, Francisco Elejabarrieta, Pierre Vergès y Silvia Valencia. El libro es un ejemplo de la variedad investigativa que se presenta en las representaciones sociales, con diversas aproximaciones teóricas y metodológicas, pero con un objetivo común: el conocimiento y comprensión de la realidad social presente en cada contexto. 
Tania Rodríguez incluye en esta obra el texto titulado "Sobre el estudio cualitativo de la estructura de las representaciones sociales" (2007), en el que presenta inicialmente un panorama general de la teoría de las representaciones sociales con sus definiciones, enfoques e investigadores, y después centra su atención en el estudio pormenorizado de las posibilidades de analizar la estructura de las representaciones sociales mediante la perspectiva de las metodologías cualitativas. Para esto, asume el enfoque estructural, corriente de investigación que se fundamenta en la Teoría del Núcleo Central de Jean Claude Abric, la cual establece que la estructura de una representación está conformada por un núcleo central y por elementos periféricos. Aunque este enfoque privilegia el uso del método experimental, Rodríguez hace una contribución al debate sobre los métodos que se deben utilizar en la investigación de las representaciones sociales, una discusión sobre la que todavía no existe consenso.

De acuerdo con Banchs (2000), Brasil es el país de Suramérica que más investigaciones ha realizado en el campo de las representaciones sociales, destacándose como principales líneas temáticas la salud, educación y medioambiente. Como investigadores relevantes se pueden mencionar los nombres de Ángela Arruda, Claritza Prado de Souza, Mary Jane Spink, Marcos Reigota, Celso Pereira de Sá, Nascimento Schulze, entre otros.

Ángela Arruda fue la primera investigadora latinoamericana formada bajo la dirección de Moscovici y Jodelet (Banchs, 2007). Es profesora de la Universidad Federal de Rio de Janeiro y sus trabajos investigativos se centran principalmente en salud, medioambiente y estudios de género. En Representaciones sociales y cultura en el pensamiento ambientalista brasileño, Arruda (2000) analiza las representaciones hegemónicas sobre la naturaleza y las etnias que han existido en Brasil en diferentes períodos históricos: la Colonia, el nacimiento de la nación y la proclamación de la República. Las modificaciones que han tenido estas representaciones a lo largo del tiempo se explican por su naturaleza dinámica e histórica (Rouquette, 1994).

Banchs (2007) reseña el trabajo de Arruda, La representación de la salud en un barrio de bajos recursos económicos en Campiña Grande, Paraiba (Arruda, 1994), en el que explora las representaciones de salud y enfermedad, así como sus prácticas sociales en una comunidad marginal. En este trabajo se destaca la reivindicación que hace la investigadora de la sabiduría popular, evidenciada en las prácticas de prevención y manejo de enfermedades, en la concepción integral de la salud que unifica mente y cuerpo, y en el control 
de las enfermedades hasta ciertos límites permisibles mediante el uso de la medicina casera o popular, de tal forma que solo cuando estas estrategias no funcionan las personas acuden al sistema de salud institucional. La autora plantea una reflexión muy valiosa acerca de la importancia del conocimiento de las prácticas para generar posibilidades de intervención, reflexión que define una posición ética frente a la realidad y motiva la investigación al respecto. En este caso particular, el conocimiento de la realidad social puede ser muy útil para la transformación de políticas de salud tendientes al mejoramiento de la calidad de vida de las comunidades.

Marcos Antonio Dos Santos Reigota es considerado un erudito en estudios sobre educación y medioambiente. Cursó estudios de Filosofía en Brasil y posteriormente realizó su doctorado en Educación en Bélgica. Es profesor del programa de Postgrado en Educación de la Universidad de Sorocaba e investigador del Consejo Nacional de Investigación Científica de Brasil. Su tesis de doctorado, Las representaciones sociales del medioambiente y las prácticas pedagógicas cotidianas de los docentes de ciencia en Sao Paulo, Brasil (1990), se considera el punto de partida de las investigaciones en representaciones sociales sobre el medioambiente.

En Las representaciones sociales como práctica pedagógica cotidiana de la educación ambiental, Reigota (2010) formula una propuesta política y pedagógica que se fundamenta en la Teoría de las Representaciones Sociales de Moscovici, en la Pedagogía Dialógica de Freire y en la Educación Ambiental como alternativa política. Para Reigota, las posibilidades de intervención que ofrece el estudio y conocimiento de las representaciones sociales presentes en los contextos socioculturales de nuestro continente son una razón suficiente para el desarrollo de prácticas pedagógicas en educación ambiental cuyo objetivo sea identificar las representaciones de los estudiantes, estimular el pensamiento crítico, el debate y la toma de posiciones ante la realidad. Su propuesta concreta se refiere a una pedagogía dialógica orientada hacia la transformación de la realidad social.

Celso Pereira de Sá, profesor de Psicología en la Universidad del Estado de Rio de Janeiro, ha desarrollado estudios en los campos de las representaciones sociales, comportamientos sociales y en memoria social e histórica. Sus aportes al campo de las representaciones sociales son bastante significativos, pero desafortunadamente la difusión de sus estudios ha tenido dificultades por la escasa aceptación del idioma portugués en el ámbito académico internacional, como comenta Lobato (2011). 
Se destaca su libro $A$ construção do objeto de pesquisa em representações sociais (1998) con prólogo de Jodelet, dirigido esencialmente a los investigadores que desean conocer el proceso de construcción de un objeto de estudio en representaciones sociales, así como la estrategia teórico-metodológica apropiada. Merece resaltarse la claridad con la que Sá explica cuál debe ser el enfoque teórico que debe utilizarse en la investigación (procesual, estructural, toma de posición) dependiendo de los objetivos planteados. Asimismo, en el capítulo dedicado a los métodos, se describen las diferentes posibilidades de aproximación con sus limitaciones y se hacen recomendaciones bibliográficas de interés. En conclusión, una obra valiosa desde lo pedagógico y didáctico que fue el resultado de diálogos e intercambios entre el profesor Sá y sus estudiantes de pregrado y postgrado (Lobato, 2011).

Claritza Prado de Sousa pertenece al Núcleo de Investigación en Representaciones Sociales en Educación del Programa de Postgrado en Psicología de la Educación de la Pontificia Universidad Católica de Sao Paulo. Sus preferencias investigativas se encuentran en el campo de los procesos educativos. En Develando la cultura escolar, Prado de Souza (2000) hace una presentación de trabajos de investigación educativa realizados en Brasil, cuya finalidad es la comprensión de los procesos socioculturales que favorecen el fenómeno de la exclusión escolar de un número significativo de jóvenes y niños. Prado de Souza indica que el objetivo de estas investigaciones es aprovechar las posibilidades que ofrece la Teoría de las Representaciones Sociales para generar conocimiento y producir la transformación de las prácticas, asumiendo de esta manera la misma visión y compromiso ético de investigadores como Arruda y Reigota.

Tal y como lo describen Pereira de Sá y Arruda (2000), en la pasada década de 1970, la Escuela de Altos Estudios en Ciencias Sociales de París (EHESS) ejercía una gran atracción en los profesionales de la psicología provenientes de Latinoamérica. Nuestro continente vivía entonces procesos sociales y políticos muy significativos, con dinámicas complejas que necesitaban ser comprendidas para generar conocimiento y estrategias para la intervención y transformación de la realidad de ese momento histórico particular. Por esta razón, la Teoría de las Representaciones Sociales se convirtió en una alternativa de gran interés para el desarrollo de estudios en las diversas áreas de aplicación de la Psicología Social.

Una de estas profesionales de la psicología era María Auxiliadora Banchs, de nacionalidad venezolana, quien se formó en el EHESS bajo la dirección de 
Serge Moscovici y Denise Jodelet, obteniendo su doctorado en el año 1979. En la actualidad es docente de Psicología Social de la Escuela de Psicología y de la Maestría en Psicología Social en la Universidad Central de Venezuela. La doctora Banchs es reconocida como una de las más grandes defensoras y difusoras de la Teoría de las Representaciones Sociales en Venezuela y América Latina; su obra investigativa incluye artículos, capítulos de libros y libros que han sido publicados en países como Venezuela, México, Brasil y España.

Representaciones sociales en Venezuela: la apuesta al cambio (Banchs y Lozada, 2000) presenta un panorama del desarrollo de la Teoría de las Representaciones Sociales en ese país. Sus autores describen un primer momento (1981-1985) caracterizado por la resistencia al cambio, debido a la hegemonía del paradigma de la psicología social estadounidense que estudiaba procesos individuales y utilizaba el método experimental para el análisis de los fenómenos sociales y humanos. Sin embargo, un acontecimiento que favoreció la acogida de la teoría fue el de los seminarios orientados por Denise Jodelet en 1982 y 1983 en la Universidad Central de Venezuela (Banchs, 2001). En el período de 1986-1992 se produjo la difusión y consolidación de la teoría, inicialmente con el desarrollo de estudios en forma de tesis de licenciatura y maestría en diferentes centros académicos; posteriormente se comenzaron a realizar estudios de campo específicos.

Un listado de investigadores venezolanos destacados en el estudio de las representaciones sociales incluye los nombres de Mireya Lozada, Elisa Casado, Cari Salonge, Francisco Rodríguez, María Cristina Parra, Gladys Villaroel, Alexis Romero, Luz Pargas, Carmen García, Maruja Alruiz, Marú León, Jesús Canelón y Milagros García (Banchs, 2001).

Desde el punto de vista metodológico, existe un predominio de las investigaciones que utilizan métodos cualitativos orientados hacia la comprensión de significados; son muy pocos, en efecto, los estudios que utilizan métodos estadísticos o el método experimental. Otra característica que merece resaltarse es la ausencia de investigaciones teóricas, siendo una excepción los trabajos de Banchs y Lozada. Las áreas temáticas de mayor interés son la salud, el género, problemáticas sociales, política, educación y cultura (Banchs y Lozada, 2000).

Por último, entre los años 1993-1999, se produjo el cuestionamiento de la teoría de las representaciones sociales por parte del socio-construccio- 
nismo, una perspectiva postmoderna de la psicología social que formuló una crítica conjunta en contra de la teoría de las representaciones sociales, el marxismo, el psicoanálisis y el estructuralismo, e indica la necesidad de su deconstrucción. Frente a este panorama, la propuesta de Banchs (2001) consiste en desarrollar un proceso de autorreflexión y revisión de los fundamentos epistemológicos de la teoría, tarea a la que se ha dedicado hasta la fecha presente con una serie de publicaciones en las que presenta su contribución al fortalecimiento y reivindicación como alternativa heurística para el desarrollo de la investigación social. Dentro de esta producción académica e intelectual, se pueden mencionar: Desconstruyendo una desconstrucción: lectura de lan Parker a la luz de los criterios de Parker y Shotter (Banchs, 1994); Construccionismo Social y Representaciones Sociales. Algunos puntos de encuentro y desencuentro (Banchs, 1996); Modernidad, Posmodernidad y Representaciones Sociales (Banchs, 1998); Aproximaciones procesuales y estructurales al estudio de las representaciones sociales (Banchs, 2000); La Psicología Social como práctica político-ética. Reflexiones en torno a la arista subjetiva de las Representaciones Sociales (Banchs, 1999).

Argentina, como los países latinoamericanos antes reseñados, también ha sido un terreno fértil para el florecimiento de la investigación en representaciones sociales. De nuevo, los campos de aplicación de la teoría son muy diversos, cubriendo las temáticas de ciencia y saber académico, comunidades humanas, etapas del desarrollo humano, estudios de género, educación, trabajo, problemáticas sociales y políticas.

En el campo de las representaciones sociales de la ciencia se destacan los trabajos realizados por Liliana Lacolla, del Centro de Formación e Investigación en Enseñanza de las Ciencias de la Universidad de Buenos Aires.

Su artículo "Representaciones sociales: una manera de entender las ideas de nuestros alumnos" (Lacolla, 2005), es una propuesta teórica para el mejoramiento de la enseñanza como consecuencia de la identificación y comprensión de las preconcepciones que construyen los estudiantes sobre los conceptos de la ciencia. En su tesis de doctorado "La representación social que los estudiantes poseen acerca de las reacciones químicas y su incidencia en la comprensión del concepto de cambio químico", Lacolla (2012) determina la estructura de la representación social de los estudiantes acerca de las reacciones químicas y analiza la forma cómo los modelos mentales previos inciden en la construcción de los campos conceptuales de la ciencia química, revistiendo una enorme importancia en el proceso de aprendizaje. 
El trabajo de Lacolla ha servido como referente para otros investigadores como Claudia Mazzitelli, quien forma parte del Consejo Nacional de Investigaciones Científicas y Técnicas (CONICET) y es profesora titular de la Universidad Nacional de San Juan. En "Las actitudes de los alumnos hacia las Ciencias Naturales, en el marco de las representaciones sociales, y su influencia en el aprendizaje" (Mazzitelli y Aparicio, 2009), se realiza un estudio descriptivo para identificar las actitudes asociadas a las representaciones de la ciencia que están presentes en los estudiantes de escuelas de la provincia de San Juan.

"Representaciones acerca de la enseñanza y el aprendizaje de las Ciencias durante la formación docente inicial" (Mazzitelli, 2012), por su parte, es un estudio descriptivo que utiliza una combinación de métodos cualitativos y cuantitativos para identificar las representaciones sociales de los futuros profesores de Física y Química, estudiantes de la Universidad Nacional de San Juan. El objetivo central de la investigación consiste en identificar la estructura de la representación (núcleo central y elementos periféricos) y el análisis de su incidencia en los procesos de enseñanza-aprendizaje de la ciencia.

María Matilde Balduzzi es docente e investigadora del Departamento de Psicología y del Núcleo de Estudios Educacionales y Sociales de la Facultad de Ciencias Humanas de la Universidad Nacional del Centro de la Provincia de Buenos Aires. Su línea de investigación corresponde al campo educativo, en el cual ha desarrollado diversos trabajos fundamentados en las representaciones sociales. En su trabajo, "Representaciones sociales de estudiantes universitarios y relación con el saber", Balduzzi (2011) expone los resultados de una investigación desarrollada con estudiantes universitarios, en la que identifica sus representaciones sociales del saber, la enseñanza y el aprendizaje. Sus fundamentos teóricos son diversos, comprendiendo a Moscovici, Jodelet, Abric, Moliner y Doise, mientras que en el diseño metodológico considera técnicas cualitativas como la entrevista semiestructurada y la observación.

Se trata de un estudio significativo en la comprensión de las representaciones sociales del saber de los estudiantes universitarios que confirma la interacción existente entre las concepciones presentes en los individuos y su contexto sociocultural. Para la autora, los resultados promueven una reflexión sobre la universidad y sus significados, y en especial sobre las estra- 
tegias pedagógicas y didácticas que pueden conducir a la transformación de las prácticas actuales.

Es conveniente destacar el valioso aporte teórico realizado por investigadores argentinos de las representaciones sociales como Irene Vasilachis, José Antonio Castorina y Alicia Barreiro. Irene Vasilachis se desempeña como investigadora principal del CONICET y del Centro de Estudios e Investigaciones Laborales (CEIL-PIETTE) y es considerada una especialista en el Análisis de Discurso. Sus intereses investigativos se encuentran en la epistemología, la creación mediática y política de representaciones sociales, y en la pobreza y conflictos sociales, en los cuales adopta una perspectiva multidisciplinaria que incluye el derecho, la sociología y la lingüística (Discurso y Sociedad, 2015).

Entre sus obras más destacadas se encuentra La construcción de representaciones sociales: el discurso político y prensa escrita (Vasilachis, 1997), con prólogo de T. van Dijk, en el que utiliza el análisis sociológico-lingüístico del discurso para demostrar cómo la conjunción del discurso del poder político junto a la acción de los medios de comunicación, contribuyen a naturalizar posiciones ideológicas particulares entre los individuos de una sociedad, enfrentando y deslegitimando de esta manera los desafíos de grupos que reclaman sus derechos y reivindicaciones sociales y políticas. El estudio se centra en el período histórico comprendido entre 1991 y 1996, durante el cual se realizaron reformas en la legislación laboral con el fin de someter a la Argentina a los programas de ajuste económico propuestos por el capitalismo neoliberal.

En su otra obra Pobres, pobreza, identidad y representaciones sociales, Vasilachis (2003) presenta una serie de estudios realizados con personas pobres de la ciudad de Buenos Aires y en la que propone una nueva epistemología, "la epistemología del sujeto conocido", definiendo un modo particular de acceder al conocimiento que privilegia al sujeto investigado y que se fundamenta en la igualdad esencial. De nuevo utiliza la metodología del análisis sociológico-lingüístico del discurso para comprender la forma cómo se han construido las representaciones sociales de la pobreza a través de los medios de comunicación y su influencia en la constitución de subjetividades e identidades entre los individuos de los contextos socioeconómicos más deprimidos. 
José Antonio Castorina, profesor de Psicología y Epistemología Genética en la Facultad de Filosofía y Letras de la Universidad de Buenos Aires, es autor de una numerosa producción que incluye libros, capítulos de libros y artículos referidos a temas como psicología genética, psicología del conocimiento social de los niños y epistemología de la psicología del desarrollo educacional. Se destacan sus aportes a la teoría de las representaciones sociales y su contribución en el debate epistemológico generado en torno a su consideración como enfoque investigativo en el ámbito de las ciencias sociales.

En Las representaciones sociales: problemas teóricos y desafios educativos (Castorina y Kaplan, 2003) se describen las críticas formuladas a la teoría en cuestión, especialmente la que se refiere a la falta de rigor conceptual y a la inconsistencia de sus bases epistemológicas. De acuerdo con los autores, las representaciones sociales son transversales a otras disciplinas como la psicología cognitiva, la antropología, la sociología y la lógica natural; además, se presenta una enorme complejidad para definir un concepto en el que intervienen componentes psíquicos y sociales y se repasan los diferentes argumentos formulados por Moscovici en la defensa del estatuto teórico de las representaciones sociales. Posteriormente, se realiza un análisis de la relación existente entre la psicología social y la psicología del desarrollo de los conocimientos sociales para concluir que tales disciplinas poseen bases epistemológicas y ontológicas comunes y por lo tanto se propone un espacio común para la investigación social en el que se supera la división individuo-sociedad, espacio que sería de gran utilidad para enfrentar desafíos educativos presentes en el aprendizaje de los escolares.

Las representaciones sociales y su horizonte ideológico. Una relación problemática de Castorina y Barreiro (2006) constituye una exploración de dos categorías conceptuales: la ideología y las representaciones sociales, a través de una comparación crítica que tiene en cuenta el conocimiento de sentido común, el conocimiento científico y las estructuras de dominación. El análisis realizado por Castorina y Barreiro permite concluir que en su origen social, las ideologías apuntan hacia lo general, es decir, son cosmovisiones; en tanto que las representaciones sociales se orientan hacia objetos específicos. De esto se desprende que las representaciones se construyen sobre el trasfondo de una ideología que abarca la totalidad de los objetos sociales que poseen un significado. Otra conclusión interesante para diferenciar estos dos conceptos surge del análisis de sus relaciones con el poder: en el caso de la ideología, el poder es un elemento determinante de su conformación, mientras que en la génesis de una representación no intervienen las relaciones de poder 
y dominación. En síntesis, se trata de un aporte relevante para la caracterización, comprensión y diferenciación de dos categorías conceptuales y sus relaciones con el conocimiento de sentido común, la ciencia y el poder.

\section{CONCLUSIÓN}

La propuesta investigativa de la Teoría de las Representaciones Sociales ha logrado una aplicación significativa en el estudio y comprensión de la realidad social y humana. Su objeto de estudio es amplio y diverso: psicoanálisis, ciencia, salud y enfermedad, cuerpo humano, infancia, relaciones entre grupos humanos, educación, género, inteligencia, problemáticas sociales y políticas, entre otros. Tópicos estos de gran relevancia en los estudios latinoamericanos inspirados en esta teoría.

El carácter transversal de la noción de representación social permite la integración con disciplinas como psicología, antropología, sociología e historia y, por lo tanto, el desarrollo de investigaciones orientadas hacia la comprensión de realidades complejas que se transforman constantemente. Como afirma Jodelet (2011):

La perspectiva de las representaciones sociales ha encontrado un eco muy favorable en los dominios de investigación caracterizados por la complejidad de los fenómenos o sistemas a los que se refieren, su estrecha dependencia en relación al devenir de la sociedad global, la multiplicidad de niveles a partir de los cuales pueden ser abordados: cultural, político, institucional, organizacional, interindividual, individual. (p.3)

En el caso particular de Latinoamérica, a través de este estudio se pueden evidenciar los aportes de la teoría de las representaciones sociales al campo de las ciencias sociales y la educación. Es visible el interés de los investigadores en diversos problemas atinentes a la educación y la pedagogía, así como en temas del medioambiente y la salud, y en la comprensión de nuestra realidad social.

La Teoría de las Representaciones Sociales nace en el año 1961, pero solo hasta 1980 se inicia el desarrollo de trabajos investigativos en Europa. En dicho continente existe un predominio de las investigaciones orientadas por el enfoque estructural y se destacan países como el Reino Unido, Austria, Francia e Italia. La expansión de la teoría hacia el continente americano fue precedida por la formación de psicólogos sociales latinos en la Escuela de Altos 
Estudios en Ciencias Sociales de París (EHESS), liderada por Serge Moscovici y Denise Jodelet durante la década 1970-1980. Además, la compleja realidad social, política, económica y cultural de Latinoamérica se constituyó en un objeto de estudio para los investigadores de las Ciencias Sociales.

En este sentido, Jodelet (2000) reconoce que Latinoamérica ha sido una tierra fértil para el desarrollo de investigaciones en representaciones sociales. Y este trabajo intelectual lleva implícita una dimensión ética de gran valor que se manifiesta en el respeto y reconocimiento de la realidad del sujeto investigado y ante todo en la postura axiológica asumida por el investigador ante la realidad humana:

Con relación a la ética podemos reintroducir la problemática de la creación social, del imaginario y de la utopía. En este sentido, la confrontación de la práctica de los investigadores en Europa y América Latina es instructiva. En el viejo continente -donde los investigadores están ante el reto de una influencia paradójica: la del pesimismo de la posmodernidad, en la que el porvenir está ausente; o la del control ejercido por una visión todavía positivista y pesada de nuestra práctica científica- queda poco espacio para una visión anticipadora sobre la vida social. En el nuevo continente, al contrario, más orientado hacia el cambio, la investigación está cargada de deseo, de proyección, de ideal y de utopía. Estos aspectos creativos son favorecidos en el espacio cultural de los países del nuevo mundo. (Jodelet, 2000, p.27).

Por otra parte, en América Latina, la mayoría de las investigaciones han asumido el enfoque procesual o clásico, rasgo que supone una identidad local en el uso de la teoría, y permite explorar al menos dos vetas: el interés de los investigadores criollos por develar nuestras representaciones para entender el "ontos" suramericano; y la creatividad como un elemento alimentador de nuevas metodologías y nuevas técnicas de aproximación e intervención en las comunidades.

Otro rasgo esencial que devela este artículo es la postura ética, debido a que el objetivo es aprovechar las posibilidades que ofrece la Teoría de las Representaciones Sociales para generar conocimiento sobre la realidad y producir la transformación de las prácticas.

Tanto en Venezuela como en el resto de América Latina, observamos que dentro de la psicología social como disciplina, el quehacer de investigadores 
y académicos, en general, no se ha orientado hacia la producción de conocimientos teóricos sino, más bien, hacia la producción de conocimientos sobre problemas de la realidad social, a la luz de diferentes perspectivas teóricas. El ejercicio profesional de los psicosociólogos en América Latina, a diferencia de Europa y Estados Unidos, tiene un fuerte sentido de compromiso social. Este asume un carácter ético político que implica tanto la comprensión como la posibilidad de transformación de la realidad social que estudiamos (Banchs \& Lozada, 2000, p.89).

En síntesis, el presente texto permite ubicar ciertas tendencias en el uso de la teoría de las representaciones sociales como un importante recurso para la investigación social, psicosocial y educativa. Con un interés por el enfoque comprensivo, América Latina viene aportando nuevas lecturas sobre el tema, en específico, su marcado foco como plataforma para la intervención y la transformación social.

A la fecha presente, sin embargo, teóricos como Rouquette (2010) afirman que la Teoría de las Representaciones Sociales está pasando por una fase de retracción científica que se manifiesta en la falta de innovación teórica y metodológica, la desaparición de revistas especializadas y laboratorios de investigación, así como en la disminución de las investigaciones en este campo.

Nota de autores: Este artículo se origina en dos investigaciones del macroproyecto titulado "Representaciones sociales sobre conflicto armado, paz y cultura de paz" y, de manera concreta, en la investigación "Reconocimiento y cultura para las paces: un estudio desde la vida cotidiana de estudiantes universitarios".

\section{REFERENCIAS}

Abric, J. (2001). Prácticas sociales y representaciones. México: Ediciones Coyoacán.

Alfonso, N. (2006). Representaciones sociales y prácticas investigativas. Revista Itinerantes, (4), 119-130. Recuperado de: http://revistas.udem.edu.co/index.php/ opinion/article/view/575

Araya, S. (2002). Las representaciones sociales: ejes teóricos para su discusión. Cuaderno de Ciencias Sociales, 127. FLACSO (Facultad Latinoamericana de Ciencias Sociales). Recuperado de: http://unpan1.un.org/intradoc/groups/ public/documents/ICAP/UNPAN027076.pdf

Arruda, A. (1994). La representación de la salud en un barrio de bajos recursos económicos en Campiña Grande, Paraiba. Anthropos: Boletín de información y documentación, (44), 97-101. Recuperado de: https://dialnet.unirioja.es/servlet/ articulo? codigo $=279697$

Arruda, A. (2000). Representaciones sociales y cultura en el pensamiento ambientalista brasileño. Develando la cultura estudios en representaciones sociales. México: Facultad de Psicología, UNAM. 
Balduzzi, M. M. (2011). Representaciones sociales de estudiantes universitarios y relación con el saber. Espacios en blanco. Serie indagaciones, 21(2), 183-218. Recuperado de: http://www.scielo.org.ar/pdf/eb/v21n2/v21n2a02.pdf

Banchs, M. (1998). Modernidad, Posmodernidad y Representaciones Sociales. Simpósio Internacional sobre Representacões Sociais: questões epistemológicas. Textos para a discussão. Brasil: CEPAL.

Banchs, M. A. (1994). Desconstruyendo una desconstrucción: lectura de lan Parker (1989) a la luz de los criterios de Parker y Shotter (1990). Papers on Social Representations, 3(1), 52-74. Recuperado de: http://www.psych.Ise.ac.uk/Psr/ PSR1994/3_1994Banch.pdf

Banchs, M. A. (1996). Construccionismo Social y Representaciones Sociales. Algunos puntos de encuentro y desencuentro. Revista AVEPSO, XIX (2), 1-10. Recuperado de: http://www.scielo.org.co/scielo.php?script=sci_arttext\&pi$d=S 1692-715 \times 2008000100003$

Banchs, M. A. (2000). Aproximaciones procesuales y estructurales al estudio de las representaciones sociales. Papers on Social Representations, 9(3), 1-3. Recuperado de: http://www.psych.Ise.ac.uk/psr/PSR2000/9_3Banch.pdf

Banchs, M. A. (1999). La psicología social como práctica político ética: reflexiones en torno a la arista subjetiva de las representaciones sociales. Psicología Social, 12(1/2), 32-53. Recuperado de: http://pepsic.bvsalud.org/scielo.php?script=sci_ arttext\&pid=S1794-99982008000200002

Banchs, M. A. (2001). Jugando con las ideas en torno a las representaciones sociales desde Venezuela. Fermentum, 11(30), 11-32. Recuperado de: http://www.saber. ula.ve/bitstream/123456789/20683/1/articulo1.pdf

Banchs, M. A. (2007). Entre la ciencia y el sentido común: representaciones sociales y salud. En Rodríguez, García (Coordinadoras), Representaciones sociales. Teoría e investigación. México: Universidad de Guadalajara.

Banchs, M. A. \& Lozada, M. (2000). Representaciones sociales en Venezuela: la apuesta al cambio. En D. Jodelet y A. Guerrero (Ed), Develando la cultura. Estudios en representaciones sociales, 89-108. México, DF: UNAM. Recuperado de: http:// saber.ucv.ve/jspui/handle/123456789/13838

Castorina, J. A. \& Barreiro, A. (2006). Las representaciones sociales y su horizonte ideológico. Una relación problemática. Boletín de Psicología (Valencia), (86), 7-25. Recuperado de: https://www.uv.es/seoane/boletin/previos/N86-1.pdf

Castorina, J. A. \& Kaplan, C. (2003). Las representaciones sociales: problemas teóricos y desafíos educativos. En J. Castorina (Comp), Representaciones sociales. Problemas teóricos y conocimientos infantiles (p.9-27), Barcelona: Gedisa.

Clèmence, A. \& Lorenzi-Cioldi, F. (2005). Dinámica de la representación. De las representaciones mentales a las representaciones sociales de los grupos. Trayectorias, 7(18), 64-78. Recuperado de: http://www.redalyc.org/pdf/360/36021230010. pdf

Colombia Digital (2014). ¿Qué es Internet invisible o Internet profunda? Recuperado de: https://colombiadigital.net/actualidad/articulos-informativos/ item/6296-que-es-internet-invisible-o-internet-profunda.html

Doise Doise, W. (1991a). Las representaciones sociales: presentación de un campo de investigación. Anthropos. Boletín de información y documentación, (27), 196-207. Recuperado de: http://www.comunicacionysociedad.cucsh.udg.mx/index.php/ comsoc/article/view/1792

Doise, W. \& Mugny, G. (1991b). Psicología social experimental: percepción intelectual de un proceso histórico: veinte años de psicología social en Ginebra. Revista de 
Documentación Científica de la Cultura, (124), 8-32. Recuperado de: https:// archive-ouverte.unige.ch/unige:15763

Doise, W., Clèmence, A. \& Lorenzi-Cioldi, F. (1993). The quantitative analysis of social representations. USA: Taylor \& Francis.

Doise, W., Clèmence, A. \& Lorenzi-Cioldi, F. (2005). Representaciones sociales y análisis de datos. México: Instituto Mora.

Domínguez, S. (2012). Significados de la Ciencia en estudiantes universitarios. Aproximaciones a las representaciones sociales de la ciencia, del científico y de la actividad científica. México: Universidad de Guadalajara, CUCSH.

Flament, C. (2001). Estructura, Dinámica y Transformación de las Representaciones Sociales. En J. Abric (Ed.), Prácticas sociales y representaciones. (pp.33-52). México: Ediciones Coyoacán.

Guerrero, A. (2000). La noción de igualdad en la cultura mexicana. En D. Jodelet., y A. Guerrero (Ed), Develando la cultura. Estudios en representaciones sociales (pp.187-219). México: Facultad de Psicología/UNAM.

Guimelli, C. \& Rouquette, M. (1992). Contribution du modèle associatif des schemes cognitifs de base à l'analyse structurale des représentations sociales. Bulletin de Psychologie, 45(1), 196-202. Disponible en http://www.bulletindepsychologie. net/vente/achat/produit_details.php?id=194

Jodelet, D. (2000). Representaciones sociales: Contribución a un saber sociocultural sin fronteras. En D. Jodelet., y A. Guerrero (Ed), Develando la cultura. Estudios en representaciones sociales (pp.7-30). México, DF: UNAM.

Jodelet, D. (2011). Aportes del enfoque de las representaciones sociales al campo de la educación. Espacios en blanco. Serie indagaciones, 21(1), 133-154. Disponible en http://www.scielo.org.ar/scielo.php?script=sci_arttext\&pid=S1515-94852011000100006

Jodelet, D. \& Guerrero, A. (2000). Develando la cultura: estudios en representaciones sociales. México: Universidad Nacional Autónoma de México, Facultad de Psicología.

Jovchelovitch, S. (2001). Social Representations, Public Life and Social Construction. En K. Deaux \& G. Philogéne (Ed) Representations of the Social (pp.165-182). Oxford: Blackwell.

Jovchelovitch, S. (2007). Knowledge in Context. Representations, Community and Culture. London: Routledge.

Lacolla, L. (2005). Representaciones sociales: una manera de entender las ideas de nuestros alumnos. Revista ieRed: Revista Electrónica de la Red de Investigación Educativa, 1(3), 1-17. Disponible en http://biblioteca.universia.net/html_bura/ ficha/params/title/representaciones-sociales-manera-entender-ideas-alumnos/ id/5787856.html

Lacolla, L. H. (2012). La representación social que los estudiantes poseen acerca de las reacciones químicas y su incidencia en la construcción del concepto de cambio químico. (Tesis doctoral) Universidad de Burgos, Burgos.

Lobato, A. (2011). Reseña del libro A construção do objeto de pesquisa em representações sociais, del profesor Celso Pereira de Sá. Magis. Revista Internacional de Investigación en Educación, 3(6), 461-464. Disponible en http://revistas.javeriana. edu.co/index.php/MAGIS/article/view/3552

Lorenzi-Cioldi, F. (1994). Les androgynes [The androgynes]. Paris: Presses Universitaires de France.

Lorenzi-Cioldi, F. (1996). Psychological androgyny: a concept in search of lesser substance. Towards the understanding of the transformation of a social repre- 
sentation. Journal for the Theory of Social Behaviour, 26(2), 137-155. https://doi. org/10.1111/j.1468-5914.1996.tb00526.x

Markovà, I. (2000). Amédée or how to get rid of it: Social representations from a dialogical perspective. Culture \& Psychology, 6(4), 419-460. https://doi. org/10.1177/1354067X0064002

Markovà, I. (2003). Dialogicality and Social Representations. The Dynamics of Mind. Cambridge: Cambridge University Press.

Markovà, I. \& Wilkie, P. (1987). Representations, concepts and social change: The phenomenon of AIDS. Journal for the Theory of Social Behaviour, 17(4), 389-409. https://doi.org/10.1111/j.1468-5914.1987.tb00105.x

Mazzitelli, C. A. (2012). Representaciones acerca de la enseñanza y el aprendizaje de las Ciencias durante la formación docente inicial. Revista de Curriculum y formación del profesorado. 16(3), 1-16. Recuperado de https://recyt.fecyt.es/ index.php/profesorado/article/view/43442

Mazzitelli, C. A. \& Aparicio, M. T. (2009). Las actitudes de los alumnos hacia las Ciencias Naturales, en el marco de las representaciones sociales, y su influencia en el aprendizaje. REEC: Revista electrónica de enseñanza de las ciencias, 8(1), 193-215. Disponible en https://dialnet.unirioja.es/servlet/articulo?codigo=3041509

Moliner, P. (1989). Validation expérimentale de l'hypothèse du noyau central des représentations sociales. Bulletin de Psychologie, 41(1), 759-762. Disponible en http://psycnet.apa.org/record/1990-76150-001

Moliner, P. (1993). ISA: I'induction par scenario ambigü. Une méthode pour l'étude des représentations sociales. Revue Internationale de Psychologie Sociale, 6(2), 7-21. Disponible en http://www.europhd.net/bibliographic-item/isa-linduction-par-sc\%C3\%A9nario-ambigu-une-m\%C3\%A9thode-pour-1\%C3\%A9tude-des-representations

Mugny, G. \& Carugati, F. (1985). L'intelligence au pluriel. Cousset: DelVal.

Pereira de Sá, C. (1998). A construção do objeto de pesquisa em representações sociais. Rio de Janeiro: Editora da Universidade do Estado do Rio de Janeiro, EdUERJ.

Pereira de Sá, C. \& Arruda A. (2000). O estudo das representações sociais no Brasil. Revista de Ciências Humanas, Florianópolis: EDUFSC, Edição Especial Temática, 19, 11-31. Disponible en https://periodicos.ufsc.br/index.php/revistacfh/article/ view/24121

Prado de Souza, C. (2000). Develando la cultura escolar. En D. Jodelet., A. Guerrero. (comp.). Develando la cultura. Estudios en Representaciones sociales. México: Universidad Nacional Autónoma de México.

Rateau, P. \& Monaco, G. L. (2013). La Teoría de las Representaciones Sociales: orientaciones conceptuales, campos de aplicaciones y métodos (la Theorie des Representations Sociales: orientations conceptuelles, champs d'applications et methodes). Revista CES Psicología, 6(1), 22-42. Disponible en http://revistas.ces. edu.co/index.php/psicologia/article/view/2615

Reigota, M. (1990). Les representations sociales de l'environnement et les pratiques pedagogiques quotidiennes des professeurs de sciences à São Paulo-Brésil. (Tesis de Doctorado). Universidad Católica de Lovaina, Bélgica

Reigota, M. (2010). Las representaciones sociales como práctica pedagógica cotidiana de la educación ambiental. En Investigación y educación ambiental (pp.71-79), Bogotá: Universidad Distrital Francisco José de Caldas. 
Rodríguez, T. (2007). Sobre el estudio cualitativo de la estructura de las representaciones sociales. En Rodríguez, García (Coordinadoras), Representaciones sociales. Teoría e investigación. México, Universidad de Guadalajara, 157-188.

Rodríguez, T. \& García, M. (2007). Representaciones sociales. Teoría e investigación. México: Editorial CUCSH-Universidad de Guadalajara.

Rouquette, M. L. (2010). La teoría de las representaciones sociales hoy: esperanzas e impases en el último cuarto de siglo (1985-2009). Polis, 6(1), 133-140. Disponible en http://www.scielo.org.mx/scielo.php?script=sci_arttext\&pid=S1870-23332010 000100006

Rouquette, M., L. (1994). Sur la connaissance des masses. Essai de psychologie politique. Grenoble: Presses Universitaires de Grenoble.

Vasilachis, I. (1997). La construcción de representaciones sociales. Discurso político y prensa escrita. Un análisis sociológico, jurídico y lingüístico. Barcelona: Gedisa.

Vasilachis, I. (2003). Pobres, pobreza, identidad y representaciones sociales. Barcelona: Gedisa.

Vasilachis de Gialdino, I. (2015). Discurso y Sociedad. Recuperado de: http://www. dissoc.org/dissoc/comite/vasilachis/

Wagner, W. (1994). The fallacy of misplaced intentionality in social representation research. Journal for the Theory of Social Behavior, 24(3), 243-266. https://doi. org/10.1111/j.1468-5914.1994.tb00255.x

Wagner, W. \& Elejabarrieta, F. (1994). Representaciones sociales. En Morales, J. Francisco (Ed). Psicología Social. Madrid: UNED-McGraw-Hill.

Wagner, W., Duveen, G., Farr, R., Jovchelovitch, S., Lorenzi-Cioldi, F., Markovà, I. \& Rose, D. (1999). Theory and method of social representations. Asian Journal of Social Psychology, 2(1), 95-125. https://doi.org/10.1111/1467-839X.00028

Wagner, W., Elejabarrieta, F. \& Lahnsteiner, I. (1995). How the sperm dominates the ovum -objectification by metaphor in the social representation of conception. European Journal of Social Psychology, 25(6), 671-688.

Wagner, W., Valencia, J. \& Elejabarrieta, F. (1996). Relevance, discourse and the 'hot' stable core of social representations. A structural analysis of word associations. British Journal of Social Psychology, 35(3), 331-351. https://doi. org/10.1111/j.2044-8309.1996.tb01101.x

Wagner, W. \& Hayes, N. (2005). Everyday Discourse and Common Sense. The Theory of Social Representations. Hampshire: Palgrave. 